\title{
Bold steps to tackle resistance
}

\author{
The continued rise in antimicrobial resistance shows that the current efforts to combat the crisis \\ are not enough. More global leadership from policymakers and the industry is needed.
}

\section{Cf economic aspects are at the heart of a possible solution}

Despite substantial efforts, untreatable 'superbugs' are on the rise and are spreading fast. So, what does it take to tackle the resistance crisis? In a new Collection of Review articles we highlight how the global scientific and public health communities as well as governmental and regulatory bodies are focusing on key areas to address the threat posed by antimicrobial resistance.

Extended-spectrum $\beta$-lactam-resistant and carbapenem-resistant Klebsiella pneumoniae are critical public health threats. Besides the high prevalence of health-care-associated opportunistic K. pneumoniae infections, severe community-acquired infections are on the rise; in Malawi, $>75 \%$ of $K$. pneumoniae bloodstream infections are multi-drug resistant, a particularly worrying trend in such a resource-limited country, where detection, surveillance, treatment and infection control are challenging. Genomics are imperative in understanding and tracking the evolution and spread of resistant bacteria such as K. pneumoniae in the hospital or community settings, and in informing public health (Holt and colleagues).

Drug-resistant fungal infections such as Candida auris pose an important clinical problem. Currently, only three classes of antifungal agents are used, and emerging resistance to even one drug class greatly limits therapeutic options. The problem is exacerbated by fungal tolerance, a phenomenon that is not detected with traditional diagnostic tests and might account for the discordance between overall treatment outcome and infection by apparently drug-susceptible fungal strains (Berman and Krysan).

New and improved diagnostics are not only needed to detect tolerance - rapid antimicrobial susceptibility testing (AST) systems are also crucial in guiding therapy decisions. van Belkum and colleagues assess new, rapid AST systems, urging that "it is important to spend less time on small, incremental improvements to existing technology, but rather to strive for substantial advances". Rapid ASTs can help preserve currently available antimicrobials by ensuring appropriate treatment.

Since the 1980s, no new class of antibiotics that has made it into the clinic has been discovered, and, troublingly, no new class of antibiotics effective against Gram-negative bacteria has been developed since the 1960s. Theuretzbacher and colleagues review the clinical pipeline of antibacterial agents. Developing a new drug is costly and time-consuming, and owing to some degree of pre-existing cross-resistance, especially in Gram-negative critical priority pathogens, the scope and expected life span of these new drugs, which are mainly derivatives of most major known chemical and functional classes, seems limited. This is a disheartening outlook of the antibiotic pipeline, and certainly the discovery of new drug targets and innovation become more pressing. The urgent need for new drugs with novel modes of action is also underscored by the emergence and spread of antimalarial resistance. Fidock and colleagues argue that pursuing resistance drivers, such as the chloroquine-resistance transporter of Plasmodium falciparum, as drug targets constitutes a promising approach to restore existing drugs. Theuretzbacher and colleagues take stock of the global preclinical antibacterial pipeline, discussing 407 projects, including novel scientific concepts and non-traditional approaches. Yet, innovative approaches to drug discovery carry high translational risks, and most of those projects are led by small-sized or medium-sized enterprises, which may not have the same resources as big pharmaceutical companies to support the projects pre-launch and postlaunch (for example, Achaogen and Melinta recently filled for bankruptcies), and large companies are leaving the antibiotic development space all together.

Although the scientific challenges may perhaps be addressed, key factors contributing to the decline in antibiotic drug discovery and development are indeed economic. In a Viewpoint article, Årdal, Balasegaram, Laxminarayan, McAdams, Outterson, Rex and Sumpradit argue that we need new business models for drug development and new approaches to develop, share and preserve antibiotics - a tall order. Encouragingly, the UK initiated a trial to test a 'subscription' style payment model, delinking payment from antibiotic sales volume to incentivize the pharmaceutical industry. In a Comment, Nathan proposes a provocative plan, involving three cooperative, interdisciplinary, international councils that control the development, financing and use of new drugs.

Certainly, there is no lack of awareness of the problems and hurdles, nor of plans and strategies to tackle the resistance crisis. What is needed are bold steps from policymakers and the industry to make headway. The economic aspects are at the heart of a possible solution, which will ensure antimicrobial discovery and development, and global access to antibiotics. 